\title{
Detecting the Kondo screening cloud around a quantum dot
}

\author{
Ian Affleck ${ }^{1,2}$ and Pascal Simon ${ }^{2}$ \\ ${ }^{1}$ Canadian Institute for Advanced Research and ${ }^{2}$ Department of Physics and Astronomy, University of British Columbia, \\ Vancouver, B.C., Canada, V6T $1 Z 1$
}

\begin{abstract}
A fundamental prediction of scaling theories of the Kondo effect is the screening of an impurity spin by a cloud of electrons spread out over a mesoscopic distance. This cloud has never been observed experimentally. Recently, aspects of the Kondo effect have been observed in experiments on quantum dots embedded in quantum wires. Since the length of the wire may be of order the size of the screening cloud, such systems provide an ideal opportunity to observe it. We point out that persistent current measurements in a closed ring provide a conceptually simple way of detecting this fundamental length scale.
\end{abstract}

The almost trivial looking problem of a single quantum spin interacting with a gas of otherwise non-interacting electrons has attracted much attention from condensed matter theorists for decades [1]. In particular, it played an important role in the development of scaling and renormalization group ( $\mathrm{RG}$ ) ideas. While the "bare" dimensionless coupling constant, $\lambda$ is small, the low energy behavior of the system is described by an infinite effective Kondo coupling. This implies that a magnetic impurity makes a large contribution to the resistivity of the metal at low temperatures, corresponding to the "unitary limit". The characteristic energy scale at which the effective coupling constant becomes large defines the Kondo temperature:

$$
T_{K} \approx D e^{-1 / \lambda}
$$

where $D$ is the bandwidth and we have set Boltzmann's constant to one. Associated with this energy scale is a fundamental length scale:

$$
\xi_{K}=\hbar v_{F} / T_{K}
$$

where $v_{F}$ is the Fermi velocity. For a typical metal exhibiting the Kondo effect this length scale can be of order .1 microns or larger. A simple picture of the meaning of this length scale is that a single electron occupying a wave-function extended over this distance forms a spin singlet with the impurity spin (which is assumed to have $S=1 / 2$ here). Physical quantities such as the Knight shift can be expressed in terms of universal scaling functions of $r / \xi_{K}$ where $r$ is the distance from the impurity 2,3]. Recent numerical simulations [2] have explicitly confirmed such scaling behavior. Nevertheless, no experiment has ever directly detected this fundamental length scale. The reason for this is perhaps mainly that this scale is too large.

While the original Kondo effect led to a large resistivity at low $T$, a quite different manifestation of the same physics was predicted in a slightly different experimental configuration, corresponding to a large transmission coefficient 4.57. A quantum dot can act like an $\mathrm{S}=1 / 2 \mathrm{im}-$ purity due to the Coulomb blockade. By adjusting a gate voltage on the dot, it can be arranged that the groundstate of the dot has an odd number of electrons, with an unpaired spin pointing up or down, and that the energy cost to add or remove an electron is relatively large. When this quantum dot is connected to leads with weak tunneling matrix elements, the transmission coefficient of the dot is small at most temperatures. This system is essentially equivalent to the Kondo model with the dimensionless coupling constant being of order $t^{2} / \epsilon_{0} D$ where $t^{\prime}$ is the tunneling matrix element, $\epsilon_{0}$ is the energy to add or remove an electron from the dot and $D$ is the bandwidth of the leads. Now the growth of this effective coupling constant at low temperatures is manifested by the dot exhibiting a transmission coefficient close to 1 at temperatures below $T_{K}$. Recent developments in nanotechnology have made it possible to observe various aspects of this "transmission Kondo effect" in the last few years [6].

Since Kondo temperatures in these experiments are generally considerably smaller than $1^{0} K$ (and can be tuned via the gate voltage, $\epsilon_{0}$ ) the Kondo length scale is expected to be of order 1 micron or larger. Again, the simple picture is that an electron in the leads, occupying a wave-function spread out over this length scale, screens the spin of the quantum dot. This new experimental realization of the Kondo effect seems to offer new opportunities to measure the screening cloud. In some of these experiments [9], the quantum dot is connected to thin leads (quantum wires) of length of order $1 \mathrm{mi}-$ cron, which, in turn are connected to macroscopic leads further from the dot. One might hope to see a change in behavior when the length of the quantum wire leads becomes comparable to $\xi_{K}$. (The dot itself is generally much smaller than the screening cloud.) Indeed, this notion of suppression of the Kondo effect by a finite size is central to much of the theoretical work on the problem; see especially the nice interpretation of Wilson's numerical renormalization group calculations by Nozières [10]. However, the situation in these experiments is complicated by the fact that the screening cloud may also occupy the macroscopic parts of the leads. While devices can be envisioned [11] that might get around this problem, our goal here is to study the conceptually simplest way of probing screening cloud physics in such systems.

We consider a quantum dot in a closed mesoscopic circular ring [12 14. In this geometry the screening cloud 
is "trapped" in the ring and cannot escape into macroscopic leads. The experimental challenge is to measure the transmission amplitude through the dot without attaching leads to the ring. This could be done by measuring the persistent current in the ring induced by a magnetic flux. Such persistent current experiments have been performed recently on micron sized rings not containing a quantum dot 15,16]. We shall assume here that the ring contains no other impurities besides the quantum dot. It is encouraging in this regard to note that recent experiments, with macroscopic leads attached, observed perfect conductance $\left(2 e^{2} / h\right)$ through a quantum dot [9]. We only consider $T=0$ here (effectively $T<<T_{K}$, $\left.T<<\hbar v_{F} / L\right)$ although our results could be extended straightforwardly to higher $T$. One might expect that the persistent current, as a function of the flux, $\Phi$ penetrating the ring, will be much different when the screening cloud is much smaller than the circumference, $L$, of the ring than when it is much larger. We will argue below that, when $\xi_{K}<<L$, the persistent current is that of a perfect ring with no impurity, of order of magnitude $j \propto e v_{F} / L$. On the other hand, when $\xi_{K}>>L, j L$ becomes much smaller, vanishing as a power of the bare Kondo coupling. In general, $j(\Phi) L$ is a universal scaling function of $\xi_{K} / L$, in the usual scaling limit of the Kondo model (i.e. at small Kondo coupling and large ring size compared to the lattice constant). The functional form of $j(\Phi)$ crosses over from a saw-tooth for $\xi_{K}<<L$ to a sine function at $\xi_{K}>>$.

We begin with the standard tight-binding Anderson model for the quantum dot-quantum wire system [4, 5 , $H=H_{0}+H_{\text {int }}$ with

$$
\begin{gathered}
H_{0}=-t \sum_{j \leq-2}\left(c_{j}^{\dagger} c_{j+1}+h . c .\right)-t \sum_{j \geq 1}\left(c_{j}^{\dagger} c_{j+1}+\text { h.c. }\right) \\
H_{i n t}=-t^{\prime}\left[c_{0}^{\dagger}\left(c_{-1}+c_{1}\right)+\text { h.c. }\right]+\epsilon_{0} c_{0}^{\dagger} c_{0}+U n_{0 \uparrow} n_{0 \downarrow} .
\end{gathered}
$$

We then pass to the Kondo limit, $t^{\prime}<<-\epsilon_{0}, U+\epsilon_{0}$, where the dot is singly occupied and virtual tunneling to the neighboring sites (at $j= \pm 1$ ) lead to a spin-exchange interaction:

$$
H_{\text {int }}=J\left(c_{-1}^{\dagger}+c_{1}^{\dagger}\right) \frac{\vec{\sigma}}{2}\left(c_{-1}+c_{1}\right) \cdot \vec{S}
$$

The Kondo coupling is $J=2 t^{2}\left[-\epsilon_{0}^{-1}+\left(U+\epsilon_{0}\right)^{-1}\right]$. The dimensionless Kondo coupling appearing in Eq. (1) is $\lambda=4 J \sin ^{2} k_{F} / \pi v_{F}$, where $k_{F}$ is the Fermi momentum. The zero temperature persistent current is given by $j=-(e / \hbar) d E_{0} / d \alpha$ where $E_{0}$ is the groundstate energy, and a magnetic flux, $\Phi=(\hbar c / e) \alpha$ is applied to the ring, corresponding to modifying phases of hopping terms so that the sum of phases is $\alpha$. We consider a ring of $L$ sites. To calculate $j$ in the large $L$ limit, we may linearize the dispersion relation around the Fermi surface, leading to simple formulas for the electron propogator in the time domain and thus facilitating perturbation theory in $\lambda$.
Ignoring corrections down by $1 / L$, and working to $O\left(\lambda^{2}\right)$ for an even number of electrons, $N$ and to $O\left(\lambda^{3}\right)$ for odd $N$, we find:

$$
\begin{aligned}
j_{e}(\alpha)= & \frac{3 \pi v_{F} e}{4 L}\left\{\left[\sin \tilde{\alpha}\left[\lambda+\lambda^{2} \ln (L c)\right]\right.\right. \\
& \left.+(1 / 4+\ln 2) \lambda^{2} \sin 2 \tilde{\alpha}\right\}+O\left(\lambda^{3}\right) \\
j_{o}(\alpha)= & \frac{3 \pi v_{F} e}{16 L} \sin 2 \alpha\left[\lambda^{2}+2 \lambda^{3} \ln \left(L c^{\prime}\right)\right]+O\left(\lambda^{4}\right),
\end{aligned}
$$

for $N$ even and odd respectively, where $c$ and $c^{\prime}$ are constants of $\mathrm{O}(1)$ which we have not determined and:

$$
\begin{aligned}
& \tilde{\alpha}=\alpha \quad(N / 2 \text { even }) \\
& \tilde{\alpha}=\alpha+\pi \quad(N / 2 \text { odd }) .
\end{aligned}
$$

Importantly, $j(\alpha, \lambda, L) L$, to the order we have worked, is a function only of $\alpha$ and the renormalized Kondo coupling at scale $L: \lambda_{\text {eff }}(L)=\lambda+\lambda^{2} \ln L+\ldots$. That this should be true exactly, in the scaling limit, follows from standard RG arguments. (See, for example [3].) Since the current is conserved it may be calculated at an arbitrary point in the ring, far from the quantum dot. Therefore it has vanishing anomalous dimension since the interactions all take place near the origin and anomalous dimensions vanish for all operators far from the impurity. Therefore the dimensionless quantity $L j$ can only depend on the effective coupling at scale $L$ or equivalently on the ratio $\xi_{K} / L$. In particular, this implies that the perturbative result becomes valid at small $L / \xi_{K}$ where $\lambda_{e f f}(L)$ is small. The corrections to this scaling form are suppressed by factors of $a / L$, where $a$ is the lattice constant (which we have set to 1 ).

To calculate $j$ at large $L / \xi_{K}$, we may use the fact that $\lambda_{e f f} \rightarrow \infty$ and that $j$ is a (universal) characteristic of the infrared fixed point. Thus we may obtain it from the Kondo Hamiltonian of Eq. (4) by setting $J \rightarrow \infty$. In this limit one electron is trapped in the symmetric orbital on sites $\pm 1,(1>+\mid-1>)$. The low energy effective Hamiltonian is simply a non-interacting tightbinding model

$$
\begin{aligned}
H_{\text {low }}= & -t \sum_{j \leq-3}\left(c_{j}^{\dagger} c_{j+1}+\text { h.c. }\right)-t \sum_{j \geq 2}\left(c_{j}^{\dagger} c_{j+1}+\text { h.c. }\right) \\
& -\frac{t}{\sqrt{2}}\left(-c_{-2}^{\dagger} c_{a}+c_{a}^{\dagger} c_{2}+\text { h.c. }\right)
\end{aligned}
$$

where $c_{a} \equiv\left(c_{1}-c_{-1}\right) / \sqrt{2}$. This model exhibits resonant transmission at $k=\pi / 2$, corresponding to half-filling, due to particle-hole symmetry. The persistent current in such a non-interacting, scattering model, is completely determined by the transmission amplitude at the Fermi surface [17]. Thus, in the half-filled case, we obtain the persistent current for an ideal ring. We should take into account an effective shift of $\alpha$ by $\pi$ due to the reversed sign for the hopping term between sites -2 and 0 in Eq. 
(7) and also the fact that the number of low energy electrons is $N-2$. These two effects on the persistent current cancel so that it is exactly the same as in original model of Eq. (3) with $U=0, t^{\prime}=t$. Thus we obtain, after simply adding the contributions of spin up and down electrons, the persistent current for $L>>\xi_{K}$ :

$$
\begin{aligned}
& j_{e}(\alpha)=-\frac{2 e v_{F}}{\pi L}[\tilde{\alpha}-\pi], \quad(N \text { even }) \\
& j_{o}(\alpha)=-\frac{e v_{F}}{\pi L}([\alpha]+[\alpha-\pi]) \quad(N \text { odd }) .
\end{aligned}
$$

It can easily be proven, for $L>>1$ and arbitrary $\xi_{K} / L$, that $j_{e}$ for the two cases of $N / 2$ even or odd are related by a $\pi$ shift of $\alpha$, i.e. that a single functon $j_{e}(\tilde{\alpha})$ describes both cases. Thus the persistent current, for $L>>1$ and $\lambda<<1$ is given by two universal scaling functions $j_{e} L\left(L / \xi_{K}, \tilde{\alpha}\right)$ and $j_{o} L\left(L / \xi_{K}, \alpha\right)$. We have calculated these functions in the limits of small and large arguments in Eqs. (5) and (8). They are shown in FIG. 1 .
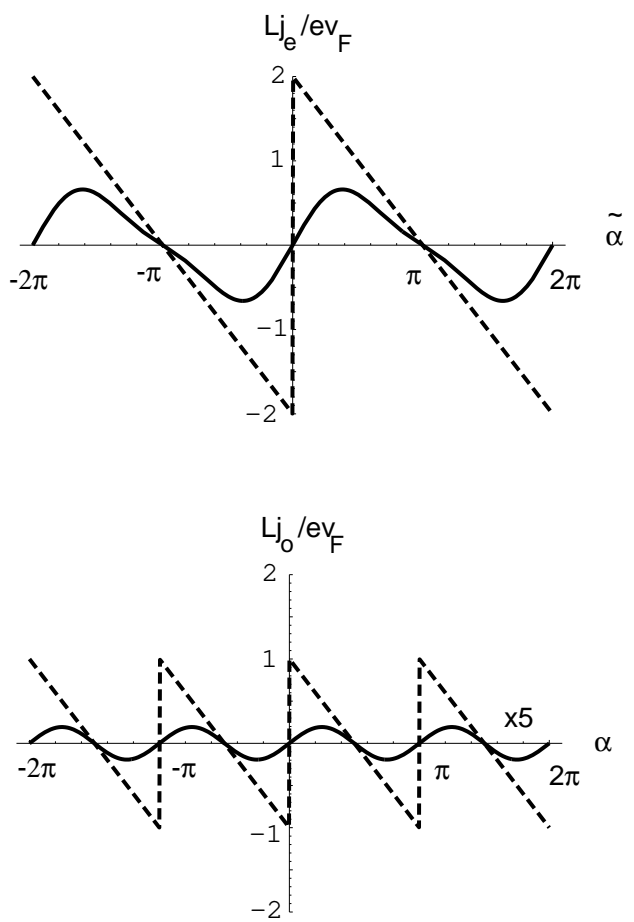

FIG. 1. Persistent current vs. flux for an even or odd number of electrons for $\xi_{K} / L \approx 50$ (solid line) and for $\xi_{K} / L<<1$ (dashed line). $j_{o}$ is multiplied $\times 5$ at $\xi_{K} / L=50$ for visibility. The solid lines are obtained from Eq. (5) using the effective coupling $\lambda(L) \approx 1 / \ln \left(\xi_{K} / L\right)$.

We expect the current at intermediate $\xi_{K} / L$ to interpolate smoothly between these limits. Note that the persistent current has the same sign, for all $\alpha$, at both small and large $\xi_{K} / L$, as in the non-interacting limit of the Anderson model, thus respecting the Leggett conjecture. Also note that $j_{o}(\alpha)$ has period $\pi$, while $j_{e}(\tilde{\alpha})$ has period $2 \pi$ for both large and small $\xi_{K} / L$. (We suspect that these properties are true in general, but have not been able to prove this.) There is a large parity effect, especially at large $\xi_{K} / L$, where the current is much smaller for $N$ odd.

In fact, the $J \rightarrow \infty$ limit of Eq. (7), only gives perfect transmission for $k_{F}=\pi / 2$, at half-filling; in general the transmission amplitude is $T(k)=\sin ^{2} k$ for $H_{l o w}$. However, it is known that particle-hole symmetry breaking is an exactly marginal perturbation in the Kondo problem so that for small bare Kondo coupling, the effective particle-hole symmetry breaking remains small in the low energy limit. (See, for example [3] .) Thus we expect the persistent current to depend only weakly on electron density and gate voltage, for all $\xi_{K} / L$, in the Kondo regime for small bare Kondo coupling. This situation changes considerably if we include screened Coulomb interactions in the ring. Then particle-hole symmetry breaking becomes relevant (for repulsive interactions) and the persistent current only achieves its ideal value for $L>>\xi_{K}$ at a special resonant value of the gate voltage, $\epsilon_{0}$ [18,17.

If we take non-symmetric tunneling amplitudes from the wire to the dot, $t_{l}^{\prime} \neq t_{r}^{\prime}$, then the persistent current at $L>>\xi_{K}$ is reduced by a factor proportional to $\left[2 t_{r}^{\prime} t_{l}^{\prime} /\left(t_{r}^{\prime 2}+t_{l}^{\prime 2}\right)\right]^{2}$ but still exhibits scaling behavior.

Real quantum wires have several active channels (i.e. transverse sub-bands). However, it is reasonable to expect that one channel will have a stronger tunneling amplitude to the dot than the others. The RG equations, to third order, for the multi-channel Kondo problem are:

$$
d \lambda_{i} / d \ln l=\lambda_{i}^{2}-(1 / 2) \lambda_{i} \sum_{j} \lambda_{j}^{2}
$$

We see that if all but one of the couplings, $\lambda_{1}$, are small, while $\lambda_{1}$ is larger and positive, then we may approximate the equation for the small couplings by only the second term in Eq. (9), keeping only the $\lambda_{i} \lambda_{1}^{2}$ term. This equation then predicts that all the small couplings shrink. Meanwhile, the larger coupling grows. It can be easily seen from this equation that this behavior is generic. The impurity spin is screened by an electron from the most strongly coupled channel and the other channels decouple at low energies. Thus, for $L>>\xi_{K}$ we expect the single channel result of Eq. (8) to still apply.

We have also studied the case of a "side-coupled" quantum dot. In this case, the electrons can only hop from the dot to site 0 on the chain. The corresponding Kondo Hamiltonian is:

$$
H_{R}=-t \sum_{j=-\infty}^{\infty}\left(c_{j}^{\dagger} c_{j+1}+\text { h.c. }\right)+J c_{0}^{\dagger} \frac{\vec{\sigma}}{2} c_{0} \cdot \vec{S} .
$$

This Hamiltonian is well-known to exhibit perfect reflection ( $\pi / 2$ phase shift in the even channel) at low energies. Thus the transmission amplitude vanishes so that the persistent current is zero for $L>>\xi_{K}$. On the other hand, for small $J$, the persistent current is nearly that of 
an ideal ring. This is the inverse of the situation for the embedded dot, discussed above. We note however, that this simple model give a much less realistic description of a real experiment in the side-coupled case. In this case all of the channels should have significant transmission past the dot, for small $J$. With increasing $L$ only one channel gets a reduced current as a result of screening the impurity spin. Furthermore, if the quantum dot is represented by more than one side coupled site, at large $J$, the screening cloud can form entirely off the ring, with no reduction of the persistent current. In this situation it is far from clear what the behavior will be for small bare coupling and large $L / \xi_{K}$.

The persistent current for the embedded dot was studied in 14, by solving some approximate self-consistent equations. The results were much different than ours. In particular, the current was predicted to be much smaller for $N$ odd than for $N$ even for all $L / \xi_{K}$. We find this result only in the perturbative regime, $\xi_{K}>>L$. This appears to indicate that these self-consistent equations are not sensitive to the infrared divergences at large $L$ related to the RG flow. (We have similar disagreements on the side-coupled case also studied using the same method [19.)

The side-coupled dot was also studied in 20]. The opposite conclusion to ours was reached that the ring would exhibit a perfect persistent current, rather than zero current, at large $L / \xi_{K}$. We believe that this incorrect result arose from a misinterpretation of some excitation parameters in the Bethe ansatz solution and an invalid general formula for the persistent current which treats incorrectly the contribution to the current from electrons below the Fermi surface.

In conclusion, we have shown that the persistent current through a quantum dot in a mesoscopic closed ring depends strongly on the ratio of the screening cloud size to the ring circumference. This provides hope that the elusive Kondo screening cloud may eventually be directly measured.

\section{ACKNOWLEDGMENTS}

I. A. thanks Silvano De Franceschi and the organizers of the NATO Workshop on Size-Dependent Magnetic Scattering (Pécs, Hungary, May 2000) for stimulating his interest in this subject. This research was supported in part by NSERC of Canada.

[1] A. Hewson, The Kondo Effect to Heavy Fermions, Cambridge University Press, 1993.
[2] E. Sørensen and I. Affleck, Phys. Rev. B53, 9153 (1996).

[3] V. Barzykin and I. Affleck, Phys. Rev. B57, 432 (1998).

[4] L.I. Glazman and M.E. Raikh, JETP Lett. 47, 452 (1988).

[5] T.K. Ng and P.A. Lee, Phys. Rev. Lett. 61, 1768 (1988).

[6] D. Goldhaber-Gordon, H. Shtrikman, D. Mahalu, D. Abusch-Magder, U. Meirav and M.A. Kaster, Nature 391, 156 (1998).

[7] S.M. Cronewett, T.H. Oosterkamp, L.P. Kouwenhoven, Science 281, 540 (1998).

[8] F. Simmel, R.H. Blick, U.P. Kotthaus, W. Wegsheider, M. Blichler, Phys. Rev. Lett. 83, 804 (1999).

[9] W.G. van der Wiel, S. De Franceschi, T. Fujisawa, J.M. Elzerman, S. Tarucha and L.P. Kouwenhoven, Science, 289, 2105 (2000).

[10] P. Nozières, Proceedings of the 14th International Conference on Low Temperature Physics, (ed. M. Krusius and M. Vuorio, North-Holland, Amsterdam, 1975), Vol. 5, p. 339.

[11] S. De Franceschi, private communication.

[12] M. Büttiker and C.A. Stafford, Phys. Rev. Lett. 76, 495 (1996).

[13] V. Ferrari, G. Chiappe, E.V. Anda and M.A. Davidovich, Phys. Rev. Lett. 82, 5088 (1999).

[14] K. Kang and S.-C. Shin, preprint, cond-mat/9912399.

[15] V. Chandrasekhar, R.A. Webb, M.J. Brady, M.B. Ketchen, W. J. Gallagher and A. Kleinsasser, Phys. Rev. Lett. 67, 3578 (1991).

[16] D. Mailly, C. Chapelier and A. Benoit, Phys. Rev. Lett. 70, 2020 (1993); W. Rabaud, L. Saminadayar, D. Mailly, K. Hasselbach, A. Benoit, and B. Etienne, preprint, condmat/0007396.

[17] A.O. Gogolin and N.V. Prokof'ev, Phys. Rev. B50, 4921 (1994).

[18] C.L. Kane and M.P.A. Fisher, Phys. Rev. B46, 15233 (1992).

[19] S.Y. Cho, K. Kang, C.K. Kim and C.-M. Ryu, preprint, cond-mat/0011215.

[20] H.-P. Eckle, H. Johannesson and C.A. Stafford, preprint, cond-mat/0010101. 